\title{
Positronium and intratrack physico-chemical processes in liquids
}

\author{
V.M. BYAKOV
}

Institute of Theoretical and Experimental Physics, 117259 Moscow, Russia

\begin{abstract}
A quantitative model for the formation of $P s$ and of radiolytic hydrogen in aqueous solutions is formulated. The concentrations dependencies of the respective yields obtained are in accordance with the experimental data.
\end{abstract}

\section{Introduction}

The effects of various solutes on the Ps yield in dielectric liquids are among the most informative experimental materials needed to elucidate the mechanism of Ps formation.

A wide experimental investigation of aqueous and polar solvents solutions made by Abbe et al. (1) has lead them to the conclusion that there exist five types of variations of the ortho-Ps fraction, $I_{3}$, with solute concentration, $\mathrm{C}_{S}$ :

- a decrease of $I_{3}$ with increasing $c_{s}$ until complete inhibition :

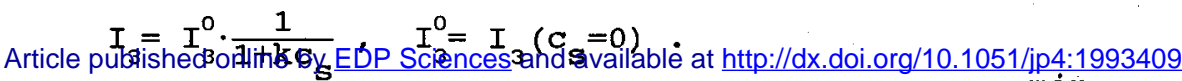

- a decrease of $I_{3}$ with $c_{S}$ until a plateau value, $I_{3}^{\text {min }}$ :

$$
I_{3}=I_{3}^{0}\left(\frac{F}{1+K c_{S}}+(1-F)\right),
$$

where $F=\left(I_{3}^{0}-I_{3}^{m i n}\right) / I_{3}^{0}$ is the fraction of Ps liable to limited inhibition.

- an increase of $I_{3}$ with $c_{s}$ up to a plateau value, $I_{3}^{\max }$ :

$$
I_{3}=I_{3}^{0} \cdot \frac{1+\left(I_{3}^{\max } / I_{3}^{0}\right) \cdot \beta C_{s}}{1+\beta C_{s}},
$$

where $k, K$ and $\beta$ are empirical constants.

- some solutes display more intricate behavior and $I_{3}$ values can be fitted using the following expressions:

$$
I_{3}=I_{3}^{0} \cdot \frac{1}{1+\mathrm{KC}_{\mathrm{S}}}\left(\frac{\mathrm{F}}{1+\mathrm{Kc}}+(1-\mathrm{F})\right)
$$

or

$$
I_{3}=I_{3}^{0} \cdot \frac{1+\left(I_{3}^{\max } / I_{3}^{0}\right) \cdot \beta C_{S}}{1+\beta c_{S}}\left(\frac{F}{1+K c_{S}}+(1-F)\right) \text {. }
$$


According to (1) all $I_{3}$ vs $c_{s}$ curves can be fitted using one of the eqs.1-5. Therefore, one of the main problems for each model of Ps formation is to derive the above equations, or some equivalent to them, on a theoretical basis.

Moreover, equations similar to eqs.1,2 describe the concentration dependencies not only of the probabilities of Ps formation but also of the decrease with $c_{s}$ of the yield of radiolytic hydrogen created under the action of ionizing radiation in aqueous solutions. This means that the mechanisms of radiolytic hydrogen formation and of Ps are similar. The aim of this report is to demonstrate that eqs.1-5 are natural consequences of the diffusion recombination model for $\mathrm{H}_{2}$ and $P s$ formation (see (2) and the references cited therein).

2. Diffusion recombination model.

According to this model, Ps and radiolytic hydrogen are formed in intratrack recombination reactions of primary chemically active products of the radiolysis of liquids : radical cations, thermalized quasi-free electrons and, of course, the de-energized positron. The reaction of $\mathrm{H}_{2}$ formation occurs in every part of the ionizing particle track (spurs, short cylindrical columns and blobs), whereas Ps is formed in the final part of the positron track, a positron blob. e creates the blob when its energy becomes less then 500 $\mathrm{eV}$ (2). Total number $\mathrm{n}_{0}$ of ion-electron pairs in the blob is $500 \mathrm{eV} /$ $17 \mathrm{eV} \approx 30$, where $17 \mathrm{eV}$ is the average energy of one ion-electron pair formation in liquid water.

The initial energies of the electrons knocked out in the positron blob are in the interval from 0 to about $100 \mathrm{eV}$. Those electrons which energy exceeds $I_{L}$, ionizing potential in the liquid phase, lose their energy very effectively because of subsequent ionizations. Thus, we may assume, that the kinetic energy of the secondary electrons is less than $I_{L}$. Following process of termalization of these secondary electrons (primarily because of excitations of inter- and intramolecular vibrations) takes, for example, in liquid water 10-20 fs. Only at the end of this process, the electrons begin to take part in reactions with radical cations and the positron.

on the contrary, electron capture by molecules is possible at substantially higher energies. Reaction cross sections, $\sigma_{s}$ ' in the gas phase frequently have one or several resonance maxima at energies about several ev. The same is true for condensed phase.

We adopt the following scheme. For energies of the electrons, higher than a threshold value for capture, $E^{*}$, only the resonance attachment reactions of the electrons with the solute take place. At lower energies, the electrons reacts with holes, the positron, traps, and possibly with solute, because of non-resonance capture.

The fraction $\varphi\left(E^{*}\right)$ of electrons escaping resonance capture by solute, and, therefore reaching energies $\mathrm{E}<\mathrm{E}$, during their slowing down is given by :

$$
\varphi\left(E^{*}\right)=\int_{0}^{E^{*}} f(E) d E+\int_{E^{*}}^{I_{L}} d E f(E) \cdot \exp \left(-C_{S} \int_{E^{*}}^{I_{L}} \frac{\sigma_{S^{\prime}}\left(E^{\prime}\right)}{\left|d E^{\prime} / d x\right|} d E^{\prime}\right) .
$$

The first term on the right hand side of eq. 6 represents the fraction of those electrons initially created with energies below $E^{*}$. The second term shows the fraction of those electrons escaped the resonance capture by the solute. In eq. $6,-d E / d x$ is the linear energy transfer for subionizing electrons and $f(E)$ is the energy spectrum of elec- 
trons arising as the result of ionizations of molecules in the positron blob, normalized to unity :

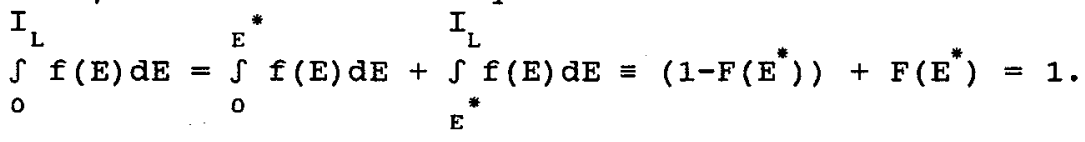

It may be seen from eq. 6 that with increasing scavenger concentration, $\mathbf{C}_{\mathbf{S}}$, the number of electrons having energies $0<\mathrm{E}<\mathrm{E}$ decreases, until a constant, nonzero value, given by the fraction $1-F\left(E^{*}\right)$ of the electrons initially created with energy within this interval. It is clear that with increasing concentration of those acceptors having cross section maximum in the thermal energy region, all knocked out electrons will be captured.

Two different approximations follow from eq.6. The first one is: $\varphi\left(E^{*}\right) \approx\left[1-F\left(E^{*}\right)\right]+F \cdot \exp \left(-C_{S} / C_{37}\right)$,

$$
1 / c_{37}=\frac{1}{|\mathrm{dE} / \mathrm{dx}|_{E=E}{ }^{*}} \int_{0}^{I_{L}} \sigma_{\mathrm{S}}(E) \mathrm{dE} \approx t_{t h} \cdot\left(\frac{2}{\mathrm{mE}^{*}}\right)^{1 / 2} \cdot \int_{0}^{I_{L}} \sigma_{S}(E) d E .
$$

Here $t_{t h}$ is the thermalization time of the subionizing electrons. The sharper the resonance of $\sigma_{s}(E)$, the more exact are eqs.8,9.

Another approximation of eq. 6 is obtained from its asymptotic expression at high values of $c_{s}: \varphi\left(E^{*}\right) \propto 1 / c_{s}$. Using this result the following interpolation between small and high $c_{S}$ values may be proposed:

$$
\begin{aligned}
& \varphi\left(E^{*}\right)=\frac{F}{1+K_{C_{S}}}+(1-F), \\
& K=\frac{1}{F\left(E^{*}\right)} \cdot \int_{E^{*}}^{I_{L}} \mathrm{dE} \cdot f(E) \int_{E^{*}}^{E} \frac{\sigma_{S^{\prime}}\left(E^{\prime}\right)}{\left|d E^{\prime} / d x\right|} d E^{\prime} .
\end{aligned}
$$

Now let us try to describe the processes occurring in spurs with the participation of the electrons with energies lower than $E$. It is convenient to adapt this description for polar liquids (water), as various experimental data concerning intratrack processes have been accumulated for aqueous solutions. In liquid water, in the end part of the positron track, the following chemical reactions take place (2) :

electron solvation :

ion-molecule reaction :

Ps formation :

ion-electron recombination :

electron capture :

hole capture :

$$
\begin{aligned}
& e^{-} \stackrel{\tau_{\text {solv }}^{-1}}{\rightarrow} e_{\text {solv }}^{-}, \\
& \mathrm{H}_{2} \mathrm{O}^{+}+\mathrm{H}_{2} \mathrm{O} \stackrel{\tau_{\text {imr }}^{-1}}{\longrightarrow} \mathrm{H}_{3} \mathrm{O}^{+}+\mathrm{OH} \text {, } \\
& \mathrm{e}^{+}+\mathrm{e}^{-} \stackrel{\mathrm{k}_{\mathrm{ep}}}{\mathrm{k}} \text { Ps, } \\
& \mathrm{e}^{-}+\mathrm{H}_{2} \mathrm{O}^{+} \stackrel{\mathrm{k}_{e h}}{\longrightarrow} \mathrm{H}_{2}, \mathrm{H}, \mathrm{H}_{2} \mathrm{O} \text {, } \\
& e^{-}+s \stackrel{k_{s}^{e}}{\rightarrow} s^{-} \text {, } \\
& \mathrm{H}_{2} \mathrm{O}^{+}+\mathrm{S} \stackrel{\mathrm{k}_{\mathrm{s}}^{\mathrm{h}}}{\longrightarrow} \text { Products. }
\end{aligned}
$$


Here $\tau_{\text {solv }}^{-1}$ is the solvation rate of the quasi-free electron and $\tau_{\text {imr }}^{-1}$ is the ion-molecule reaction rate of the radical-cation $\mathrm{H}_{2} \mathrm{O}^{+}$(hole). $\mathrm{k}_{e h^{\prime}} \mathrm{k}_{e p^{\prime}} \mathrm{k}_{\mathrm{s}}^{\mathrm{e}}$ and $\mathrm{k}_{\mathrm{s}}^{\mathrm{h}}$ are the recombination rate constants of the particles and the capture rate constants by solute, respectively. According to the concept developed here, only quasi-free ( $e^{-}$) but not solvated $\left(e_{s}\right)$ electrons contribute to the reaction of Ps formation. Starting from the prescribed diffusion method for describing inhomogeneous reactions of electrons, holes and the positron, it is possible to obtain the following equations (2) :

$$
\begin{aligned}
& \dot{n}_{e}=-k_{e h} n_{e} n_{h} / v-n_{e} / \tau_{e}, \quad n_{e}(0)=n_{0} \cdot \varphi, \\
& \dot{n}_{h}=-k_{e h} n_{e} n_{h} / v-n_{h} / \tau_{h}, \quad n_{h}(0)=n_{0}, \\
& \dot{n}_{p}=-k_{e p} n_{e} n_{p} / v, \\
& n_{p}(0)=1, \\
& v v_{0}(1+t / \tau)^{3 / 2}, \quad v_{0}=\left(2 \pi a^{2}\right)^{3 / 2}, \tau=a^{2} /(4 D),
\end{aligned}
$$

where $n_{e}$ and $n_{h}$ are the numbers of quasi-free electrons and holes in the positron blob, respectively; $n_{p}$ is the survival probability of the positron. Note that the initial number of thermalized electrons is determined by the fraction $\varphi$ of the electrons escaping resonance capture by the solute. The length a characterizes the spherical volume initially occupied by the charged particles, with diffusion coefficient D, which is approximately the same for mobile holes and thermalized electrons. We believe that the conditions of electroneutrality needed for ambipolar diffusion are fulfilled.

Four parameters define the solution of this system. They are: $\tau_{e}^{-1}=\tau_{s}^{-1}+k_{s}^{e} \cdot c_{s}$, the decay rate of quasi-free electrons because of solvation and scavenging; $\tau_{h}^{-1}=\tau_{i m r}^{-1}+k_{s}^{h} \cdot c_{s}$, the decay rate of holes through ion-molecule reaction and possible reaction with solute ; $\tau$ is the characteristic time of the blob expansion and $2 \tau / \tau_{i e r}=$ $2 \tau \cdot k_{e h} n_{0} / v_{0}$ is the ratio of the yield of all the products of the ionelectron recombination to the yield of the electrons escaping recombination with the holes.

our aim is to calculate the $\mathrm{Ps}$ and $\mathrm{H}_{2}$ formation probabilities and the relative yield, $G\left(e_{s}^{-}\right) / G^{0}$, of solvated electrons:

$$
\begin{aligned}
& P_{P s}=\int_{0}^{\infty} k_{e p} \cdot \frac{n_{e} n_{p}}{V} d t, \quad P_{H_{2}}=\frac{1}{n_{0}} \cdot \int_{0}^{\infty} k_{e h} \frac{n_{e} n_{h}}{v} d t \\
& \frac{G\left(e_{s}^{-}\right)}{G^{0}}=\frac{1}{n_{0}} \int_{0}^{\infty} \frac{n_{e}(t)}{\tau_{\text {solv }}} d t .
\end{aligned}
$$

$\mathrm{G}^{\mathrm{o}}$ is the number of ion-electron pairs created by $100 \mathrm{eV}$ energy absorption of ionizing radiation : $G=100 \mathrm{eV} / \Delta \mathrm{W} \mathrm{eV}$, where $\Delta \mathrm{W}$ is the energy needed for formation of one ion-electron pair (in water, $\Delta W \approx 17 \mathrm{eV}) \cdot \mathrm{n}_{\mathrm{o}}=\frac{500 \mathrm{eV}}{100 \mathrm{eV}} \cdot \mathrm{G}^{0} \approx 30$.

It is possible to obtain the analytical solution of this system in some particular cases only. Here we consider two of them : Ps formation in the presence of 1) an efficient hole scavenger and 2) an efficient electron scavenger. 


\section{Effects of an efficient hole scavenger}

The interactions of $\mathrm{H}_{2} \mathrm{O}^{+}$with the solute $\mathrm{S}$ may be of two kinds : 1) proton transfer from the hole to $s$, if the proton affinity of the latter exceeds that of the matrix molecule :

$$
\mathrm{H}_{2} \mathrm{O}^{+}+\mathrm{S} \rightarrow \mathrm{OH}+\mathrm{SH}^{+} \text {. }
$$

2) electron pick-off from $S$ subject to the energetic limitation that the ionization potential of $S$ is lower than the energy of neutralization of $\mathrm{H}_{2} \mathrm{O}^{+}$:

$$
\mathrm{H}_{2} \mathrm{O}^{+}+\mathrm{S} \rightarrow \mathrm{H}_{2} \mathrm{O}+\mathrm{S}^{+} \text {. }
$$

These reactions lead to the enhancement of the Ps formation probability. In the first case, the chemically active radical cation transforms into a pair of much less chemically active species ; an electroneutral radical, $\mathrm{OH}$, and an electron-even cation, $\mathrm{H}_{3} \mathrm{O}^{+}$. In the second case the mobility of the arising electron hole $s^{+}$is much less than that of the initial radical cation $\mathrm{H}_{2} \mathrm{O}^{+}$, thus diminishing the ion-electron recombination rate constant.

Let us suppose that the solute reacts very effectively only with holes, so that

$$
\mathrm{n}_{\mathrm{h}} / \tau_{\mathrm{h}} \gg \mathrm{k}_{\mathrm{eh}} \mathrm{n}_{\mathrm{e}} \mathrm{n}_{\mathrm{h}} / \mathrm{V} \text { or } \quad \tau, \tau_{\mathrm{e}^{\prime}} \tau_{\text {ier }} \gg \tau_{\mathrm{h}} \approx\left(\mathrm{k}_{\mathrm{s}}^{\mathrm{h}_{\mathrm{s}}}\right)^{-1} \text {. }
$$

In this case the expression for Ps formation probability, $\mathbf{P}_{\mathbf{P s}^{\prime}}$ is

$$
\begin{aligned}
& \mathrm{P}_{\mathrm{PS}}=\mathrm{P}_{\mathrm{PS}}^{0} \cdot \frac{1+\left(\mathrm{P}_{\mathrm{PS}}^{\max } / \mathrm{P}_{\mathrm{PS}}^{0}\right) \cdot \beta \cdot \mathrm{C}_{\mathrm{S}}}{1+\beta \mathrm{C}_{\mathrm{S}}}\left[\frac{F}{1+\mathrm{KC}_{\mathrm{S}}}+(1-\mathrm{F})\right] \\
& \mathrm{P}_{\mathrm{PS}}^{\max }=\frac{2 \tau \varphi}{\tau_{\text {ier }}}, \quad \mathrm{P}_{\mathrm{PS}}^{\circ}=\frac{2 \tau \varphi}{\tau_{\text {ier }}}\left(1-\tau_{\mathrm{h}} / \tau_{\text {ier }}\right), \quad \beta=\mathrm{k}_{\mathrm{s}}^{\mathrm{h}} \cdot \tau_{\text {ier }}
\end{aligned}
$$

This formula coincides with the empirical eq.5, found by Abbe et al. (1).

\section{Effects of an efficient electron scavenger}

The effect of an electron scavenger is obvious : it inhibits Ps formation. To obtain $\mathrm{P}_{\mathrm{Ps}}$ ' let us suppose that the scavenger concentration is so high that the electron capture rate substantially exceeds the rate of ion-electron recombination :

$$
\mathrm{n}_{\mathrm{e}} / \tau_{\mathrm{e}} \gg \mathrm{k}_{\mathrm{eh}} \mathrm{n}_{\mathrm{e}} \mathrm{n}_{\mathrm{h}} / \mathrm{v} \text { or } \tau, \tau_{h}, \tau_{\text {ier }} \gg \tau_{\mathrm{e}} \approx\left(\mathrm{k}_{\mathrm{s}}^{\mathrm{e}} \mathrm{c}_{\mathrm{s}}\right)^{-1} \text {. }
$$

In this case, $P_{P s}$ is given by

$$
\begin{aligned}
& \mathrm{P}_{\mathrm{PS}}=\mathrm{P}_{\mathrm{PS}}^{\operatorname{extr}} \cdot \frac{1}{1+\mathrm{kc}_{\mathrm{S}}}\left(\frac{\mathrm{F}}{1+\mathrm{KC}_{\mathrm{S}}}+(1-\mathrm{F})\right)=\frac{\varphi\left(\mathrm{E}^{*}\right)}{1+\frac{\tau_{\text {ier }}}{\tau_{\text {solv }}}+\tau_{\text {ier }} \mathrm{k}_{\mathrm{s}}^{\mathrm{e}} \mathrm{s}}, \\
& \mathrm{k}=\frac{\mathrm{k}_{\mathrm{s}}^{\mathrm{e}} \cdot \tau_{\text {ier }}}{1+\tau_{\text {ier }} / \tau_{\text {solv }}}, \quad \mathrm{P}_{\mathrm{PS}}^{\operatorname{extr}}=\frac{\mathrm{k}_{\mathrm{ep}} / \mathrm{k}_{\mathrm{eh}}}{1+\tau_{\text {ier }} / \tau_{\text {solv }}} .
\end{aligned}
$$

This formula also coincides with the empirical eq. 4 suggested by Abbe et al. (1). Here, $k$ is the $P$ s inhibition constant of the electron scavenger and $\mathrm{P}_{\mathrm{PS}}^{\text {extr }}$ is the Ps formation probability extrapolated to 
zero scavenger concentration. The latter value should not be identified with the real Ps formation probability in pure liquids.

The theoretical derivation of empirical eqs.4,5 satisfies our aims : eqs.1-3 can be obtained as particular cases of eqs.16,19.

In a quite similar way, the probability of formation of radioIytic hydrogen, $\mathrm{P}_{\mathrm{H}_{2}}$, can be obtained :

$$
\mathrm{P}_{\mathrm{H}_{2}}=\frac{\int_{0}^{\infty} \frac{\mathrm{k}_{\mathrm{eh}} \mathrm{e}^{\mathrm{n}_{\mathrm{h}}}}{\mathrm{v}} \mathrm{dt}}{\mathrm{n}_{0}}=\frac{\varphi\left(\mathrm{E}^{*}\right)}{1+\frac{\tau_{\text {ier }}^{\mathrm{sp}}}{\tau_{\text {solv }}}+\tau_{\text {ier }}^{\mathrm{sp}} \cdot \mathrm{k}_{\mathrm{s}}^{\mathrm{e}} \mathrm{c}_{\mathrm{s}}} .
$$

Here $\tau_{i e r}^{s p}=\left(\mathrm{k}_{e h} \mathrm{n}_{0}^{\mathrm{sp}} / \mathrm{v}_{0}^{\mathrm{sp}}\right)^{-1}$ characterizes the efficiency of ionelectron recombination averaged over all spurs along the track of an ionizing particle. It is seen that the reciprocal yield of radiolytic hydrogen increases linearly with the concentration of total inhibitor $\left(\varphi\left(E^{*}\right) \rightarrow 1\right.$ and $\left.F \rightarrow 0\right)$. The same situation stands for Ps yield.

Neglecting the difference between $\tau_{i e r}$ and $\tau_{i e r}^{\text {sp }}$, from eqs.19-21 we obtain the following simple relationship :

$$
\mathrm{P}_{\mathrm{H}_{2}}\left(\mathrm{c}_{\mathrm{s}}\right) \approx \frac{\mathrm{k}_{\mathrm{ep}}}{\mathrm{k}_{\mathrm{eh}}} \cdot \mathrm{P}_{\mathrm{Ps}}\left(\mathrm{c}_{\mathrm{s}}\right) .
$$

It means that the hydrogen and the Ps formation probabilities change proportionally to the electron acceptor concentration. Violation of this relation for some solutes could take place because these intercept radical cations as well.

Let us calculate the yield of solvated electrons, assuming that inequality 18 is fulfilled. Then, from eq.12 it follows that

$$
n_{e}(t)=n_{0} \cdot \varphi \exp \left(-t / \tau_{e}^{*}\right)
$$

where $1 / \tau_{e}^{*}=1 / \tau_{\text {solv }}+k_{s} c_{s}+1 / \tau_{\text {ier }}$. Using for $\varphi$ eq.8 and substituting eq. 23 into eq.14, we obtain :

$$
\frac{G\left(e_{s}^{-}\right)}{G^{0}}=\frac{F \cdot \exp \left(-c_{S} / c_{37}\right)+(1-F)}{1+\tau_{\text {solv }} / \tau_{i e r}{ }^{+\tau_{\text {solv }}}{ }^{\cdot k_{S} c_{S}}} \text {. }
$$

For efficient solvated electron scavengers $\mathrm{k}_{\mathrm{S}} \approx 10^{10} \mathrm{M}^{-1} \mathrm{~s}^{-1}$. In water and alcohols, for example, where $\tau_{\text {solv }}=0.5-20 \mathrm{ps}$, $\tau_{\text {solv }} \cdot k_{s} c_{s}=5 \cdot 10^{-3}-10^{-1}$, if $c_{s} \leqslant 1$ M. Therefore $\tau_{\text {solv }} \cdot k_{s} c_{s} \ll 1+$ $\tau_{\text {solv }} / \tau_{\text {ier. }}$. In this case at low $E^{*}$, when $F \rightarrow 1$, eq. 24 reduces to :

$$
G\left(e_{S}^{-}\right)=G^{0} \cdot \exp \left(-c_{s} / c_{37}\right) \text {, }
$$

which identifies with the empirical equation by Hunt et al. $(3,4)$ for the yield of solvated and localized electrons in picosecond pulse radiolysis experiment at high concentrations of acceptor.

The following consequence may be drawn from eqs.25 and 9 :

1 ) for a given acceptor, $c_{37}$ depends particularly on $v_{0}$, the energy of the quasi-free electron in liquid. Indeed, the threshold energy 
for electron capture in the gas phase, $E_{\text {gas' }}^{*}$ changes in the liquid phase to the energy $\mathrm{E}^{*}$

$$
\mathrm{E}^{*}=\mathrm{E}_{\text {gas }}^{*}-v_{0}-\left|\mathrm{P}_{-}\right|,
$$

where $P_{-}$is the polarization energy of the anion because of its interaction with the medium after electron capture. In the series of alcohols $v_{0}$ diminishes as one goes from lower to higher alcohols. Hence $\mathrm{E}^{*}$ also diminishes and $1 / \mathrm{C}_{37}$ increases, according to eq.9. In polar liquids, $v_{0}$ is about $-1 \mathrm{ev}$. In hydrocarbons, $v_{0} \approx 0 \mathrm{eV}$. Thus one may expect that some solutes being limited inhibitors in water, would be total ones in hydrocarbons.

2) for a given acceptor in solvents of different isotopic composition, like for example, light and heavy water, for which the threshold energy $\mathrm{E}^{*}$ is approximately the same, the difference in $\mathrm{c}_{37}$ is determined primarily by changes in the thermalization time of the subionizing electron.

/1/ Abbé,J.-Ch, Duplatre,G, Maddock,A,G, Talamoni,Y, Haessler, A J. Inorg. Nucl. Chem. 43(11), (1981) 2603

/2/ Byakov, V, M, Stepanov, S,V, Radiat. Phys.Chem. 41(3), (1993) 559

/3/ Lam, K, Y, Hunt, J,W, Int.J.Radiat. Phys.Chem. 7, (1975) 317

/4/ Hunt, J,W, Chase,W, J, Can.J.Chem. 55, (1977) 2080 